\title{
Elastic Instability and Contact Angles on Hydrophobic Surfaces with Periodic Textures
}

\author{
A. L. Dubov ${ }^{1,2}$, J. Teisseire ${ }^{1}$ and E. Barthel ${ }^{1}$ \\ 1 Surface du Verre et Interfaces, UMR 125 CNRS/Saint-Gobain, 39, quai Lucien Lefranc, F-93303 Aubervilliers, \\ Cedex, France \\ 2 A.N. Frumkin Institute of Physical Chemistry and Electrochemistry, Russian Academy of Sciences, 31 Leninsky \\ Prospect, 119991 Moscow, Russia
}

\author{
PACS 68.08.Bc - Wetting \\ PACS 68.03.Cd - Surface Tension \\ PACS 68.35.Np - Adhesion
}

\begin{abstract}
Surface textures are used to impart advanced wetting properties to surfaces. However understanding the surface response in relation to the nature of the texture is still a challenge. Here we have measured advancing and receding contact angles on model hydrophobic surfaces with cylindrical pillars as a function of the pillar spacing. We show that the dependances of both advancing and receding contact angles upon spacing are well accounted for by a simple model of the instability of the triple line, following the line elasticity theory by Joanny and de Gennes (J. Chem. Phys. 81 (1984) 552). This result demonstrates the prominent role of the triple line elasticity in determining the wetting properties of textured surfaces.
\end{abstract}

Introduction. - Controlling the work of adhesion of a liquid to a solid $w$ is a necessity in most areas of wetting and fluidics. This work of adhesion, which is the energy necessary to separate the liquid from the solid, is more directly measured through the contact angle $\theta_{0}$. Indeed if the surface tension of the liquid is $\gamma$, the work of adhesion is given by (Young-Dupré)

$$
w=\gamma\left(1+\cos \theta_{0}\right)
$$

In the specific case of low adhesion, $\theta_{0}$ is large, in the $150^{\circ}$ to $180^{\circ}$ range. This state of repellency has been considered in great detail because it leads to easy fluid detachment and low friction $[1,2]$. Because of the unavoidable polarisability of solid and liquid, low works of adhesion can be achieved only by partly lifting the liquid from the solid through surface roughness. This is the so-called Cassie state. The general understanding on the impact of roughness is summarized in the Cassie equation which expresses the work of adhesion of a rough surface as a function of the work of adhesion of a flat surface made out of the same material. We introduce the solid fraction $\phi \leq 1$, which is the ratio of the effectively wetted area to the apparent wetted area. The Cassie equation is the surface average of the work of adhesion and since areas not touching the surface do not contribute to the work of adhesion, it stands as

$$
1+\cos \theta=\phi\left(1+\cos \theta_{0}\right)
$$

These notions have been known for quite a long time, especially for stochastically rough surfaces [3], but the advent of microfabrication techniques has made it possible to generate a large variety of surfaces with well controlled, usually periodic, roughness. A host of recent experiments have explored the relation between roughness and work of adhesion [4-9]. Beyond the static contact angle, interest has also focused on contact angle hysteresis because it is directly related to a number of properties such as the detachment force of a drop, for example. More specifically very rough hydrophobic surfaces usually exhibit low hysteresis and under such circumstances droplets require low drag force for removal. Recent experiments have demonstrated strong impact of structure morphology on the contact angle hysteresis of hydrophobic surfaces [4,6,7].

However, quantitative understanding is far from complete. The Cassie equation is based on an equilibrium average of the work of adhesion over area. But if energy differences between neighboring configurations are larger than the thermal energy, the capacity of the Cassie equation to account for intrinsically irreversible processes should be called into doubts [10]. 
More advanced theories have been developed, where some sensitivity to local triple line conformations is introduced to inject irreversibility in the Cassie equation. Usually the validity of some rule of mixture for the wetting properties along the triple line is assumed [5,8]. One possibility is a macroscopic approach which extrapolates some local configuration energies from the measured contact angles [11]. This method does at least qualitatively account for the observed increase of the receding contact angle with decreasing pillar density. Another method focusses on local differentials of the energy landscape and this differential area (DA) model [8] has also proved successful in describing the receding contact angles for various types of textures.

However, generally speaking, the advancing contact angle remains a problem. It has consistently been shown experimentally that the advancing contact angle for microfabricated textured surfaces is nearly constant as the pillar spacing increases [4,6], although the value of the constant differs between experiments $\left(160^{\circ}\right.$ to $\left.175^{\circ}\right)$. Within the modified Cassie framework $[8,11]$, the advancing contact angle is either predicted to be $180^{\circ}$ (because the liquid progresses on air), which is too large, or predicted to change with solid fraction as per the Cassie equation, which is not the case. In summary these advanced theories fail at providing a consistent picture of the wetting properties of surfaces decorated with such sharply defined textures.

A different path has been shown by Joanny and de Gennes [12]. They proposed a theory for the hysteresis of hydrophilic surfaces with random distributions of work of adhesion. Irreversibility is connected to the local elastic instabilities of the triple line jumping on and off these pinning sites. Application of the concept to the calculation of receding contact angles (or effectively hysteresis) on hydrophobic pillar arrays has proved successful [9].

In this paper, we investigate the issue of the pinning of the triple line on hydrophobic surfaces textured with pillars in more detail. We have synthetized hydrophobic surfaces with model textures and measured advancing and receding contact angles in the Cassie state as a function of pillar spacing. The evolutions of advancing and receding contact angles with solid fraction (or more specifically pillar spacing), for constant feature size, are found to be consistent with previous experimental results. We show that a simple elastic line pinning model provides a consistent description of both advancing and receding contact angles. The asymmetry between these two cases proceeds from the nature of the boundary conditions when the deformation of the triple line becomes unstable. The results provide a simple demonstration of the prominent role of the elasticity of the triple line in the wetting properties of these surfaces.

Experiments. - Superhydrophobic surfaces were fabricated via silica sol-gel nanoimprint lithography (SSGNIL) [13]. With SSG-NIL we obtain silica structures with the simple and versatile conditions of sol-gel chemistry.
The patterns (Fig. 1) are rigid, transparent and defectfree. In a typical experiment, $4.5 \mathrm{~g}$ of methyltriethoxysilane (MTES) is mixed with $1.2 \mathrm{~g}$ of $\mathrm{HCl}(\mathrm{pH}=2)$ and stirred for 3 hours. The sol is spin coated on a smooth glass substrate. The mold is made of polydimethylsiloxane, which easily replicates masters obtained by photolithography on silicon wafers. It is applied onto the spin-coated gel film with a $50 \mathrm{~N}$ load at $50^{\circ} \mathrm{C}$ for $30 \mathrm{~min}$ followed by $90^{\circ} \mathrm{C}$ for another $30 \mathrm{~min}$. As-prepared samples were oxidized by UV-ozone treatment, hydrophobized with 1,1,2,2-tetrahydroperfluorodecyltrichlorosilane in an evacuated desiccator during 3 hours and carefully rinsed with isopropanol.

The geometry of the patterned samples was characterized by scanning electron microscopy and interference profilometry and proved to match the geometry of the masters. The patterns are cylindrical pillar arrays on square lattices. The size of the pillars is fixed (diameter $d=10 \mu \mathrm{m}$, aspect ratio 1) and the lattice parameter $d+l$ ranges from $2 d$ to $6 d$, varying the pillar density

$$
n=(d+l)^{-2}
$$

from $2.9 \times 10^{8}$ to $2.5 \times 10^{9} \mathrm{~m}^{-2}$.

The contact angle measurements were performed with water in the Cassie state. We used the volume oscillation method on a DSA100 (Krüss, Germany). During the experiment the volume was slowly oscillated in the range $7 \pm 5 \mu \mathrm{L}$ for the samples with $d+l=2 d$ to $5 d$ and in the range $5 \pm 2 \mu \mathrm{L}$ for the surface with $d+l=6 d$. For large pillar spacings the Cassie state is quite unstable and the experiments must be conducted with care.

The results are shown in Fig. 2 where normalized works of adhesion $w / \gamma$ for advancing and receding triple lines are plotted as a function of pillar spacing. Overall the results are similar to other data in the literature $[4,7,9]$. The advancing contact angle is constant with an average value $\theta_{a d v}=161^{\circ}$ i.e. a very small normalized work of adhesion (around 0.05), while the receding contact angle increases from $135^{\circ}$ to $152^{\circ}$ (and the work of adhesion decreases) when the normalized spacing $l / d$ increases from 1 to 5 .

Model. - General ideas on pinning emerged in the mid 80s when theoretical predictions were developed for the deformation of elastic lines propagating through heterogeneous energy landscapes. In the field of fracture, Rice has studied the morphology of crack fronts evolving in a bulk with heterogeneous toughness [14]. In the field of wetting, Joanny and de Gennes have calculated the morphology of a triple line propagating in a landscape of heterogeneous work of adhesion [12]. The deformation of the line in the plane of propagation results in a long range elastic field (for a crack) or corrugation of the liquid surface (for a triple line). As a result there emerges an energy term which is quadratic in the deformation. This term is viewed as a line elasticity. In fact the relations between energy heterogeneities (toughness or work of adhesion) in 


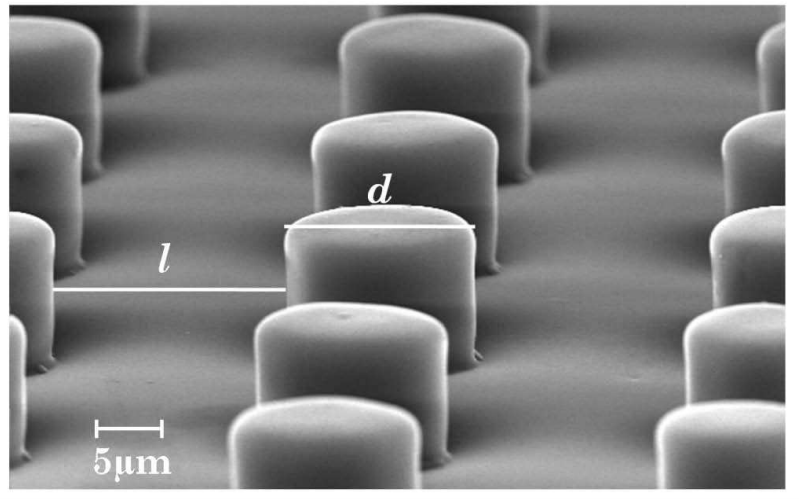

Fig. 1: SEM view of a surface textured by silica sol-gel nanoimprint lithography. Here the pillar spacing $l$ equals $10 \mu \mathrm{m}$. Various surfaces were generated where the pillar geometry is kept constant and the spacings vary from 10 to $50 \mu \mathrm{m}$.

the plane and line deformation are identical for cracks and for wetting.

The front shape can be calculated for a given pattern of energy. An extreme case appears when the energy gradient in the direction of propagation is stronger than the line stiffness, in which case local instabilities are to be expected. Series of instabilities may be triggered cooperatively resulting in avalanches. This complex response of the front shape in this so called strong pinning case leads to highly non trivial behaviour and has attracted a lot of attention in the field of statistical physics $[15,16]$.

Experimental demonstrations of the phenomena however are scarce. For fractures, the applicability of the Rice equation has been shown by direct crack front monitoring in semi transparent interfaces with simple patterns [17]. Avalanche statistics have been investigated in model heterogeneous PMMA interfaces [18]. In the field of wetting, front shapes were demonstrated to agree with the Joannyde Gennes theory and their dynamics shown to derive from viscous dissipation [19]. Statistical experiments were developed to analyze cooperative line motion for wetting surfaces [20].

To analyse the wetting properties of our surfaces (Fig. 1) we follow [12]. Compared to the homogeneous surface, the wetting energy is affected by the elastic energy barrier resulting from the presence of the pinning sites. In this scaling approach, we will assume square defects with width $d$ as in [12]. We also denote $y$ the position of the deformed liquid front, $y_{0}$ its undeformed position, $b$ the position of the edge of the defect (Fig. 3).

For a receding triple line, the wetting energy per defect is

$$
\mathcal{E}_{w}=-w d(y-b)
$$

and the elastic energy is

$$
\mathcal{E}_{e l}=\frac{1}{2} k\left(y-y_{0}\right)^{2}
$$

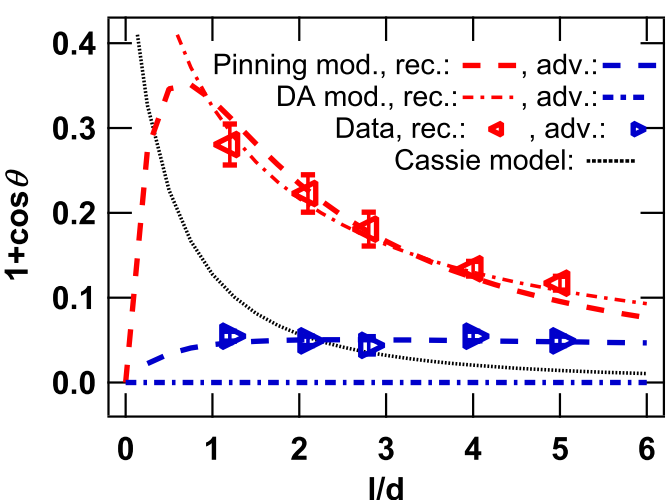

Fig. 2: Measured advancing (right triangle) and receding (left triangle) normalized work of adhesion $w / \gamma=1+\cos \theta$ as a function of pillar spacing. Error bars are mostly smaller than symbol size. A typical Cassie model (small dash) cannot account for the results. The DA model (see text; dash-dot) provides a good prediction for the receding triple line but concludes that the work of adhesion is zero for an advancing triple line. In contrast, the pinning model (dash) simultaneously accounts for advancing and receding contact angles.

Here $k$ is the elastic stiffness of the triple line characterizing the deformation energy of the liquid surface when the triple line is distorted. We assume that the line elasticity follows the same functional form as in the wetting case [12] so that

$$
k=\frac{\kappa}{\ln \left(1+\frac{l}{d}\right)}
$$

This assumption sounds reasonable: in the presence of an heterogeneity, the liquid surface deformation close to the triple line is expected to be similar for the hydrophilic and the hydrophobic cases, although the exact boundary conditions differ. Since this elastic response is ultimately due to the liquid surface tension, the constant $\kappa$ is expected to be a fraction of the liquid surface tension $\gamma$. Indeed in the hydrophilic case we have [12]

$$
\kappa=\frac{1}{2} \pi \gamma \theta_{s}^{2}
$$

where $\theta_{s}$ is the contact angle of the defect-free, wetting, surface. In the present case, the exact expression for $k$ is unknown.

Adding up Eqs. 4 and 5, we find that unstable equilibrium occurs when

$$
k\left(y-y_{0}\right)=w d
$$

At rupture the elastic energy stored in the deformation of the front is found equal to

$$
\mathcal{E}_{e l}=\frac{1}{2} \frac{(w d)^{2}}{k}
$$

This is a rupture energy for a constant force equal to $w d$, a value equal to the $90^{\circ}$ peel force of an adhesive strip of width $d$ and adhesion $w$ [21] for example. 


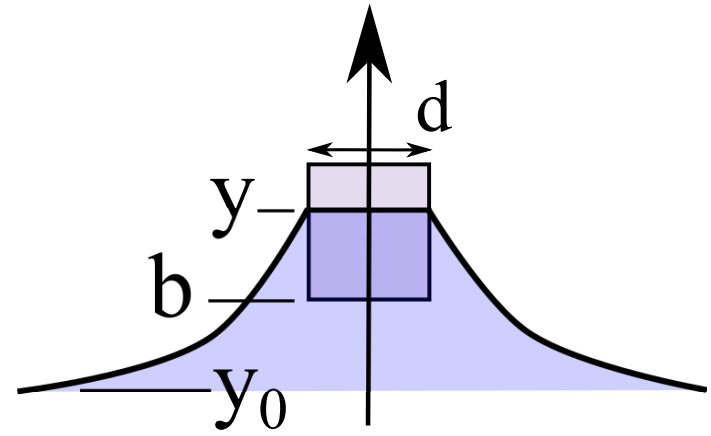

Fig. 3: Schematics of pinning for a receding triple line following Joanny and de Gennes [12]. The geometrical parameters of the instability are defect width $d$, defect position $b$, nominal triple line position $y_{0}$ and triple line deflection $y-y_{0}$. The triple line deflection at instability is determined by the competition between the peel force of the liquid from the pillar surface $w d$ and the triple line stiffness $k$.

To propagate the triple line, this elastic energy barrier has to be overcome so that the effective work of adhesion for a receding contact is

$$
\gamma\left(1+\cos \theta_{r e c}\right)=\frac{1}{2} n \frac{(w d)^{2}}{k}
$$

where $n$ is the pillar density. Following Joanny-de Gennes, this equation is obtained by summation over independent pinning sites, a process which is expected to be valid for low densities. However the result differs from the Joannyde Gennes theory because the distribution here is periodic while they assumed a random distribution to account for stochastic surfaces.

For the advancing contact angle, the line must be deformed up to the next pillar. The critical front deformation $y_{c}-y_{0}$ for instability is expected to be of the order of one pillar spacing (Fig. 4). The necessary elastic energy is evaluated as

$$
\mathcal{E}_{e l}=\frac{1}{2} k\left(y_{c}-y_{0}\right)^{2}
$$

In contrast to the receding triple line, the elastic energy stored at instability is calculated for a constant displacement (or fixed grip). Therefore we write that the critical front deformation $y_{c}-y_{0}=\lambda l$ where $\lambda$ depends on geometrical and physical parameters, but should be of the order of 1 . As a first approximation, we assume here that $\lambda$ is a constant. Finally for an advancing triple line the work of adhesion is

$$
\gamma\left(1+\cos \theta_{a d v}\right)=\frac{1}{2} n k(\lambda l)^{2}
$$

Normalizing the line stiffness by the surface tension

$$
\tilde{k}=\frac{k}{\gamma}
$$

where $k$ is given by Eq. 6, we can calculate the work of adhesion for a receding triple line by substituting Eq. 3

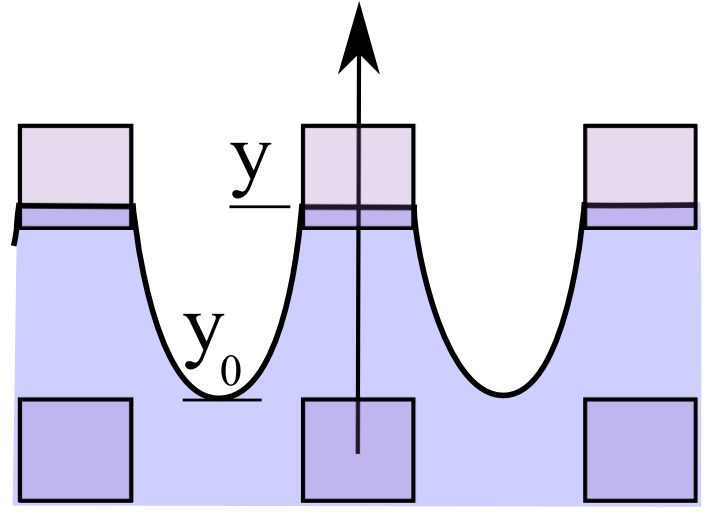

Fig. 4: Schematics of pinning for an advancing triple line following Joanny and de Gennes [12]. The deflection $y_{c}-y_{0}$ at instability is controlled by the spacing between defects in the direction of motion of the front. No attempt has been made at giving a realistic rendering of the actual deformation of the liquid surface near the front.

into Eq. 10. Normalizing the work of adhesion by $\gamma$ we obtain

$$
1+\cos \theta_{\text {rec }}=\frac{1}{2} \frac{(w / \gamma)^{2}}{\tilde{k}(1+l / d)^{2}}
$$

Similarly for an advancing triple line, by substituting Eq. 3 into Eq. 12 we obtain

$$
1+\cos \theta_{a d v}=\frac{1}{2} \tilde{k} \frac{(\lambda l / d)^{2}}{(1+l / d)^{2}}
$$

For our surfaces $d=10 \mu \mathrm{m}$ and $\theta_{0}=110^{\circ}$ which sets $w / \gamma \simeq 0.65$ according to Eq. 1. Following Eqs. 14 and 15, the receding and advancing contact angles for variable $l$ are plotted on Fig. 2 with

$$
\begin{aligned}
\tilde{\kappa} & \equiv \frac{\kappa}{\gamma}=0.11 \\
\lambda & =1.5
\end{aligned}
$$

A good agreement with the data is obtained for both receding and advancing contact angles.

We also compare the results with the so-called Cassie equation (Eq. 2 ), which predicts an effective work of adhesion proportional to density $n$ and reads

$$
1+\cos \theta=\frac{\pi}{4} \frac{w / \gamma}{(1+l / d)^{2}}
$$

From the shapes of the curves (Fig. 2) it is clear that the Cassie equation per se can account for the dependence of neither the receding nor the advancing contact angle, even assuming an effective local contact angle $\theta_{\text {eff }}$ to stand for an effective work of adhesion $w_{\text {eff }} / \gamma$.

Turning to the DA model, we calculate the differential surface texture parameter $\phi_{d}$ according to [8] and substitute this value for the solid fraction $\phi$ in the Cassie equation (Eq. 2). For circular pillars on a square array, and for 
a receding triple line, we find

$$
\phi_{d, r e c}=\frac{1}{1+\frac{l}{d}}=\sqrt{\frac{4}{\pi} \phi}
$$

which provides a good fit to the data (Fig. 2). For an advancing triple line however, the DA model predicts $\phi_{d, a d v}=0$, which does not account for the experimental results (Fig. 2).

In the pinning model, for a receding triple line (Eq. 14), the $\ln (1+l / d)$ factor originating from the elastic stiffness $\tilde{k}$ (Eqs. 6 and 13) significantly mitigates the dependence of the work of adhesion with density $n$ (Eq. 3), as noted earlier [9]. Moreover for an advancing triple line (Eq. 15), the inverse variation of the elastic pinning energy $f(l / d)=(l / d)^{2} / \ln (1+l / d)$ very nearly compensates for the variations of the density $n$, resulting in an extremely flat response for a large range of pillar spacing. As a result the product $n \times f(l / d)$ is virtually constant over the interval $1<l / d<8$ beyond which it starts to decrease more noticeably. In fact, over the range $1<l / d<8$ the product function $n \times f(l / d)$ rises from about 0.36 for $l / d=1$ to a maximum of no more than 0.41 for $l / d \simeq 2.51$ only to slowly decrease back to about 0.36 at $l / d=8$. As a result the work of adhesion for an advancing triple line, although finite, is very nearly constant for almost all practical purposes.

There is a strong contrast between the pinning and the DA models for advancing contact angles. It is worth noting that in the DA model the deformation of the triple line holds an ambiguous status: deformation is accepted but energy is minimized at fixed deformation. As a result the DA model invariably predicts an advancing contact angle equal to $180^{\circ}$ for non connected features. In contrast, the measurements consistently return values around $165^{\circ}$. This discrepancy is often ascribed to vibrations or other experimental imperfections [8]. In this paper we have emphasized that the role of line elasticity may actually be predominant in this regime. Indeed an interesting parallel may be drawn with peeling. When peeling an adhesive tape the direct work of the tension $\sigma$ is $G_{d}=h \sigma(1+\cos \theta)$ where $\theta$ is the supplement to the peel angle $\alpha$ (i.e. $\theta+\alpha=\pi$ ) and $h$ is the thickness of the tape. At equilibrium $G_{d}=w$ where $w$ is the adhesion energy of the tape; however as the peel angle $\alpha$ goes to zero $G_{d}$ goes to zero as well and this equation can no longer hold. Kendall has shown that in this case it is the (lineic) elastic energy stored in the tape $G_{e l}=h \sigma^{2} / 2 E$ which equals the work of adhesion [21]. For wetting, when the contact angle $\theta$ goes to $180^{\circ}$, the direct work of the surface tension (Eq. 1) goes to zero. The pinning theory suggests that in this case, it is the contact line elasticity which takes over to mediate between work of adhesion and contact angle, in a manner similar to peeling.

The scaling model based on the elasticity of the pinned triple line is quite powerful at accounting for the overall dependence of both advancing and receding contact angles simultaneously. In this respect note also that the values of the two free parameters are fully consistent with the assumptions of the model: the stiffness constant $\kappa$ is indeed a fraction the surface energy while the displacement parameter $\lambda$ is of the order of 1 . For an advancing triple line, the elastic energy of the line when it jumps to the next pinning site is controlled by the pillar spacing. As a result the advancing contact angle is nearly constant because the increasing elastic energy approximately cancels the decreasing number density. This quasi-constant is not zero (i.e. the contact angle is not $180^{\circ}$ ) and some energy is indeed expended in elastic deformation. In contrast, the receding contact angle is primarily controlled by the pillar size because during the instability the triple line is peeled off the top of the pillar. Hence the receding contact angle decreases roughly as the density, with a correction due to the weaker logarithmic factor which stems from triple line stiffness. For low enough densities, receding and advancing contact angles tend to converge to the same low adhesion value, resulting in low hysteresis.

Several refinements to the model can be considered. The elastic response of the triple line could be modeled more precisely and the instability conditions for both advancing and receding cases analyzed quantitatively. In particular the front shape and pillar geometry could then be taken into account accurately. This more in depth analysis can only be carried out using exact numerical solutions provided by classical fluid [22] or liquid models [23]. Then the direct contribution of the Cassie-like surface energy terms could be taken into account. Such calculations would improve the potential of the method for the design of structures with tailored wetting properties, but it is not expected that these more quantitative insights would radically alter the picture of the motion of a triple line on hydrophobic textured surfaces propounded here.

Conclusion. - Model, periodic, hydrophobic surfaces exhibit a well identified evolution of advancing and receding contact angles with solid area. A simple interpretation of the data can consistently be derived from a pinning theory which duly takes into account the elastic energy of deformation of the contact line. For an advancing triple line, the deformation occurs at a fixed displacement, and the line deformation is of the order of the pillar spacing, resulting in a nearly constant (and small) work of adhesion. In contrast line deformation proceeds at a fixed force for a receding triple line (this is similar to a peel force), giving a pronounced decrease of the work of adhesion as the pillar spacing increases. These opposite types of boundary conditions result in a strong contrast in energy barriers for advancing and receding triple lines and consistently account for the triple line response at this macroscopic level of description. 


$$
* * *
$$

We thank Elie Raphaël for interesting discussions on triple line deformation and instabilities in the context of the present experiments. We are grateful to Antoine Chateauminois for help with the elaboration of the textured surfaces. This work was supported by the National Research Agency (ANR) within the framework of the DYNALO project (project $\mathrm{n}^{\circ}$ NT09_499845).

\section{REFERENCES}

[1] Quéré D. and Reyssat M., Phil. Trans. Roy. Soc. A, 366 (2008) 1539.

[2] Stone H., Stroock A. and Ajdari A., Annu. Rev. Fluid Mech. , 36 (2004) 381

[3] Cassie A. B. D., Disc. Faraday Soc. , 3 (1948) 11.

[4] Öner D. and MCCARThy T. J., Langmuir, 16 (2000) 7777.

[5] Extrand C. W., Langmuir, 18 (2002) 7991.

[6] Dorrer C. and RüHe J., Langmuir , 22 (2006) 7652.

[7] Priest C., Albrecht T. W. J., Sedev R. and Ralston J., Langmuir, 25 (2009) 5655.

[8] Choi W., Tuteja A., Mabry J. M., Cohen R. E. and McKinley G. H., J. Colloid Interface Sci. , 339 (2009) 208.

[9] Reyssat M. and Quéré D., J. Phys. Chem. B , 113 (2009) 3906

[10] Gao L. and McCarthy T. J., Langmuir , 23 (2007) 3762.

[11] Patankar N. A., Langmuir, 26 (2010) 7498.

[12] Johnny J. F. and De Gennes P. G., J. Chem. Phys., 81 (1984) 552.

[13] Peroz C., Chauveau V., Barthel E. and SønDERGÅRD E., Adv. Mater., 21 (2009) 555.

[14] Rice J., J. Appl. Mech, 52 (1985) 571.

[15] Rosso A. and Krauth W., Phys. Rev. E , 65 (2002) 025101.

[16] Roux S., Vandembroucq D. and Hild F., European Journal of Mechanics-A/Solids, 22 (2003) 743.

[17] Dalmas D., Barthel E. and VandembroucQ D., $J$. Mech. Phys. Solids, 57 (2009) 446.

[18] Schmittbuhl J. and Maloy K., Phys. Rev. Lett. , 78 (1997) 3888.

[19] Marsh J. and Cazabat A., Phys. Rev. Lett. , 71 (1993) 2433.

[20] Moulinet S., Guthmann C. and Rolley E., Eur. Phys. J. E , 8 (2002) 437-443.

[21] Kendall K., Journal of Physics D: Applied Physics , 8 (1975) 1449.

[22] Mognetti B. M. and Yeomans J. M., Langmuir , 26 (2010) 18162

[23] Dorrer C. and RüHe J., Langmuir, 23 (2007) 3179. 